\title{
OPTIMAL FLOW \\ IN DYNAMIC NETWORKS \\ WITH NONLINEAR COST FUNCTIONS ON EDGES
}

\author{
Dmitrii Lozovanu* \\ Institute of Mathematics and Computer Science \\ Moldovan Academy of Sciences \\ 5 Academiei Str., Chisinau 2028 \\ Republic of Moldova \\ lozovanu@math.md \\ Dan Stratila \\ Faculty of Mathematics and Computer Science \\ Moldova State University \\ 60 Mateevici Str., Chisinau 2009 \\ Republic of Moldova \\ dstrat@acm.org
}

\begin{abstract}
We study the minimum-cost flow problem on dynamic networks with nonlinear cost functions that depend on time and edge flow. A general procedure for solving the problem using the time-expanded network is described. The main properties of dynamic flows on networks with concave cost functions are studied. We propose an algorithm for finding the optimal flow in networks with exactly one source; its running time is polynomial for a fixed number of sinks. Some details concerning the correctness of the algorithm and its computational complexity are discussed.
\end{abstract}

Keywords: dynamic networks, dynamic flows, flows over time, minimum-cost flows, concave cost functions.

*Supported by CRDF BGP MM2-3018.

The original version of this chapter was revised: The copyright line was incorrect. This has been corrected. The Erratum to this chapter is available at DOI: 10.1007/978-0-387-35690-7_44 


\section{Introduction and problem statement}

In this paper we study the dynamic variant of the nonlinear minimumcost flow problem on networks. This problem is a generalization of the classical static minimum-cost flow problem and is based on the dynamic network model from $[1,2]$. We consider the problem on dynamic networks with nonlinear cost functions that depend on time and edge flow. We describe a procedure for solving the problem which is based on reducing the dynamic problem to the classical minimum-cost flow problem on a time-expanded network. This procedure is based on the approach from $[1,2]$ and represents a modification of the method in [3].

We show that in the case of uncapacitated networks with concave cost functions and infinite time horizon, as well as for a wide range of fininte time bounds, there exists an optimal flow such that the edges used by the flow generate a forest. Using this result we propose a procedure that enables us to solve the dynamic problem on networks with concave cost functions on a static network of the same size. An algorithm for solving the problem on networks with one source is proposed; its running time is polynomial for a fixed number of sinks. Some details concerning the computational complexity of the algorithm and its correctness are discussed.

\subsection{Flows in static networks}

A static network $\mathcal{N}=(V, E, u, \varphi, d)$ consists of directed graph $(V, E)$, capacity function $u: E \rightarrow R_{+}$, demand and supply function $d: V \rightarrow R$ and cost function $\varphi: E \times R_{+} \rightarrow R_{+}$. Nodes with $d_{v}<0$ are called sources, nodes with $d_{v}>0$ are called sinks, and nodes with $d_{v}=0$ are called intermediate. We will denote by $V_{+}, V_{-}$, and $V_{*}$ the sets of sources, sinks, and intermediate nodes respectively.

A static flow in $\mathcal{N}$ [2] is a function $x: E \rightarrow \mathbb{R}_{+}$that satisfies for all $v \in V$ the conservation constraints:

$$
\sum_{e \in E_{-}(v)} x_{e}-\sum_{e \in E_{+}(v)} x_{e}=d_{v}
$$

Static flow $x$ is called feasible if it satisfies capacity constraints $x_{e} \leq u_{e}$ for all $e \in E$. We note that in order for a flow to exist it is necessary for demand to equal supply: $\sum d_{v}=0$.

The cost $\varphi(x)$ of static flow $x$ is defined as:

$$
\varphi(x)=\sum_{e \in E} \varphi_{e}\left(x_{e}\right)
$$


The minimum-cost static flow problem is to find a feasible flow that minimizes the objective function $\varphi(x)$.

The graph $\mathcal{G}_{x}=\left(V_{x}, E_{x}\right)$ that consists of edge set $E_{x}=\left\{e \mid x_{e}>0\right\}$ and node set $V_{x}=\left\{v \mid \exists w\right.$ such that $(v, w) \in E_{x}$ or $\left.(w, v) \in E_{x}\right\}$ is called the base graph of flow $x$ in network $\mathcal{N}$.

\subsection{Flows in dynamic networks}

In the static model defined above flow travels across edges instantaneously. However, in many practical problems flow travel across edges may take non-zero time, and the cost of flow transit may change with time. If the time factor plays a significant role, dynamic networks and dynamic flows $[1,2]$ are often a better model.

A dynamic network $\mathcal{N}=(V, E, u, \tau, \varphi, d)$ consists of directed graph $(V, E)$, capacity function $u: E \rightarrow R_{+}$, transit time function $\tau_{e}: E \rightarrow R_{+}$, demand function $d: V \rightarrow R$, and cost function $\varphi: E \times \mathbb{R}_{+} \times \mathbb{T} \rightarrow \mathbb{R}_{+}$.

The meaning of $\tau_{e}$ is that flow entering edge $e=(v, w)$ at time $t$ from node $v$ will arrive at node $w$ at time $t+\tau_{e}$. We consider the discrete time model, in which all times are integral and bounded by a time horizon $T$. The time horizon (finite or infinite) is the time until which flow can travel in the network and defines the makespan $\mathbb{T}=\{0,1, \ldots, T\}$ of time moments we consider.

To model transit costs, which may change over time, we define the cost function $\varphi_{e}\left(x_{e}(t), t\right)$, with the meaning that flow of value $\xi$ entering edge $e$ at time $t$ will incur a transit cost of $\varphi_{e}(\xi, t)$. As with the static network, nodes are categorized into sources, sinks, and intermediate. Without losing generality, we will assume that no edges enter sources or exit sinks, and that $\varphi_{e}(0, t)=0$ for all $e \in E$ and all $t \in \mathbb{T}$.

A dynamic flow is a function $x: E \times \mathbb{T} \rightarrow \mathbb{R}_{+}$that satisfies the following constraints:

$$
\begin{gathered}
\sum_{e \in E_{-}(v)} \sum_{t=\tau_{e}}^{\theta} x_{e}\left(t-\tau_{e}\right)-\sum_{e \in E_{+}(v)} \sum_{t=0}^{\theta} x_{e}(t) \geq 0, \forall v \in V_{*}, \forall \theta \in \mathbb{T} \\
\sum_{e \in E_{-}(v)} \sum_{t=\tau_{e}}^{T} x_{e}\left(t-\tau_{e}\right)-\sum_{e \in E_{+}(v)} \sum_{t=0}^{T} x_{e}(t)=d_{v}, \forall v \in V ; \\
x_{e}(t)=0, \forall e \in E, t=\overline{T-\tau_{e}+1, T}
\end{gathered}
$$

The function defines the value $x_{e}(t)$ of flow entering edge $e$ at time $t$. In order to obey the time horizon, flow must not enter an edge $e$ at time $t$ if it will have to leave the edge after time $T$, and this is ensured by constraint (4). As flow travels through the network, we allow unlimited 
flow storage at the nodes, but prohibit any deficit by constraint (2). Finally, all demands must be met, flow must not remain in the network after time $T$, and each source must not exceed its supply. Thus is ensured by constraint (3).

Dynamic flow $x$ is called feasible if it satisfies the capacity constraints $x_{e}(t) \leq u_{e}$ for all $e \in E$ and $t \in \mathbb{T}$. We note that in order for a flow to exist supply must equal demand: $\sum d_{v}=0$.

The cost $\varphi(x)$ of dynamic flow $x$ is defined as follows:

$$
\varphi(x)=\sum_{e \in E} \sum_{t \in \mathbb{T}} \varphi_{e}\left(x_{e}(t), t\right)
$$

Similarly to the static model, the dynamic minimum-cost flow problem is to find a feasible flow that minimizes the objective function $\varphi(x)$.

The graph $\mathcal{G}_{x}=\left(V_{x}, E_{x}\right)$ consisting of edge set $E_{x}=\left\{e \mid \sum_{t \in \mathbb{T}} x_{e}(t)>0\right\}$ and node set $V_{x}=\left\{v \mid \exists w\right.$ such that $(v, w) \in E_{x}$ or $\left.(w, v) \in E_{x}\right\}$ is called the based graph of dynamic flow $x$ in $\mathcal{N}$.

\section{The time-expanded network}

One of the most straightforward methods to tackle problems on a dynamic network $\mathcal{N}=(V, E, u, \tau, \varphi, d)$ is to reduce them to similar problems on a static time-expanded network $\mathcal{N}^{T}=\left(V^{T}, E^{T}, u^{T}, \varphi^{T}, d^{T}\right)$ defined as follows [2]:

$1 V^{T}:=\{v(t) \mid v \in V, t \in \mathbb{T}\}$

$2 V_{+}^{T}:=\left\{v(0) \mid v \in V_{+}\right\}$, and $V_{-}^{T}:=\left\{v(T) \mid v \in V_{-}\right\} ;$

$3 E^{T}:=\left\{\left(v(t), w\left(t+\tau_{e}\right)\right) \mid e=(v, w) \in E, 0 \leq t \leq T-\tau_{e}\right\} \cup$ $\{v(t), v(t+1) \mid v \in V, 0 \leq t<T\}$

$4 u_{e(t)}^{T}:=u_{e}$, and $\varphi_{e(t)}^{T}\left(x_{e(t)}\right):=\varphi_{e}\left(x_{e}(t), t\right)$;

$5 d_{v(t)}^{T}:=d_{v}$ for $v(t) \in V_{+}^{T} \cup V_{-}^{T}$, and $d_{v(t)}^{T}:=0$ otherwise.

If we define a flow correspondence to be $x_{e(t)}:=x_{e}(t)$ the minimum-cost flow problem on dynamic networks can be solved [2, 3, 4] by solving the static minimum-cost flow problem on the time-expanded network.

Unfortunately, for a dynamic network with $n$ nodes, and $m$ edges, we obtain a time-expanded network with $n(T+1)$ nodes, and $O(m T)$ edges. In the case of $T=\infty$, we obtain an infinite time-expanded network $\mathcal{N}^{\infty}$. Since the size of the time-expanded network depends linearly on $T$, direct use of this method does not lead to polynomial algorithms, and for large $T$ yields time-expanded networks that are not practical to work with. 
However, Fleischer and Skutella in [4] present polynomial approximation algorithms that employ "condensed" time-expanded networks which rely on a rougher discretization of time. In [3] it is shown that for an acyclic network with unit-time transit times and zero flow storage at nodes it is sufficient to consider a time-expanded network of size at most $O\left(n^{2}\right)$ nodes and $O(m n)$ edges for any $T$, finite or infinite.

In the following section we present a different approach for uncapacitated dynamic networks with cost functions that are concave with regard to flow value, and do not change over time. Relying on concavity, we reduce the problem to the minimum-cost flow problem on a static network of equal size, not the time-expanded network.

\section{Networks with concave cost functions}

Most previous work involving flow costs in dynamic networks considers linear $[4,5]$ or convex $[6,7]$ cost functions with regard to flow value. This implies that the cost of transporting one unit of flow is the same regardless of how many units are transported at a time, or that the cost per unit is rising with the total number of units. However, in many practical cases transports of larger quantities enable discounts on the price per unit. This behaviour is best modeled by cost functions concave with regard to flow value.

\subsection{Flow properties}

Given a dynamic network $\mathcal{N}=(V, E, u, \tau, \varphi, d)$, the corresponding static network $\mathcal{N}^{0}$ of $\mathcal{N}$ is obtained by discarding all time-related information: $\mathcal{N}^{0}=\left(V, E, u, \varphi^{0}, d\right)$, where $\varphi_{e}^{0}(\xi)=\varphi_{e}(\xi, 0)$.

Lemma 1. Let $\mathcal{N}$ be an uncapacitated dynamic network with cost functions concave with regard to flow and constant in time. If $x$ is a flow in $\mathcal{N}$, then $y_{e}=\sum_{t \in \mathbb{T}} x_{e}(t)$ is a flow in the corresponding static network $\mathcal{N}^{0}$ and $\varphi^{0}(y) \leq \varphi(x)$.

Proof. Note that if $\phi: R_{+} \rightarrow R_{+}$is a concave function, then $\phi(\alpha+\beta) \leq$ $\phi(\alpha)+\phi(\beta)$ for all $\alpha, \beta \in R_{+}$. Since $\varphi$ and $\varphi^{0}$ are concave with regard to flow value, we obtain $\varphi^{0}(y)=\sum_{e \in E} \varphi_{e}^{0}\left(y_{e}\right)=\sum_{e \in E} \varphi_{e}^{0}\left(\sum_{t \in \mathbb{T}} x_{e}(t)\right) \leq$ $\sum_{e \in E} \sum_{t \in \mathbb{T}} \varphi_{e}^{0}\left(x_{e}(t)\right)=\sum_{e \in E} \sum_{t \in \mathbb{T}} \varphi_{e}\left(x_{e}(t), t\right)=\varphi(x)$. Moreover, $y_{e}=$ $\sum_{t=0}^{T} x_{e}(t)=\sum_{t=\tau_{e}}^{T} x_{e}\left(t-\tau_{e}\right)$, since flow $x$ obeys constraint (4). By substituting $y_{e}$ in dynamic conservation constraint (3), we obtain the static conservation constraint (1). Therefore $y$ is a flow in $\mathcal{N}^{0}$.

This lemma relies on the fact that in uncapacitated networks each dynamic flow induces a static flow in the corresponding static network. 
While the converse is not true in the general case, it holds for flows with forest base graphs and sufficiently large time horizons.

Lemma 2. Let $\mathcal{N}$ be an infinite-horizon dynamic network with cost functions constant in time. If $y$ is a static flow in $\mathcal{N}^{0}$ such that its base graph $\mathcal{G}_{y}$ is a forest, then there exists a dynamic flow $x$ in $\mathcal{N}$ such that $\varphi(x)=\varphi^{0}(y)$.

Proof. Let $x_{(v, w)}(t)=y_{(v, w)}$ if $t=t_{v}$, and $x_{(v, w)}(t)=0$ otherwise, where:

$$
t_{v}= \begin{cases}0, & \text { if } v \in V_{+}, \\ \max \left\{t_{w}+\tau_{(w, v)} \mid(w, v) \in E_{y}\right\}, & \text { otherwise. }\end{cases}
$$

Since $\mathcal{G}_{y}=\left(V_{y}, E_{y}\right)$ is a forest, the constants $t_{v}$ are well-defined and finite. To prove that $x$ is a flow in $\mathcal{N}$, we have to show that it satisfies constraints (4), (2), and (3).

Because $T=+\infty$ it follows that $T \geq t_{v}, \forall v \in V_{y}$. Therefore, for any $e=(v, w) \in E_{y}$, we obtain $T \geq t_{w} \geq t_{v}+\tau_{e}$, hence $T-\tau_{e} \geq t_{v}$. Since $t \neq t_{v}$ implies $x_{e}(t)=0$, it follows that $x_{e}(t)=0$ for all $t_{v}>T-\tau_{e} \geq t_{v}$, hence constraint (4) is obeyed.

Definition (5) means flow starts leaving $\forall v \in V_{*} \cap V_{y}$ only after all inbound flow has arrived. Thus for $\theta<t_{v}$ we have $\sum_{e \in E_{+}(v)} \sum_{t=0}^{\theta} x_{e}(t)=0$, hence constraint (2) holds for $\theta<t_{v}$. For $\theta \geq t_{v}$ flow summed over time on any edge is the same as the flow on that edge in the static network: $\sum_{e \in E_{-}(v)} \sum_{t=\tau_{e}}^{\theta} x_{e}\left(t-\tau_{e}\right)=\sum_{e \in E_{-}(v)} y_{e}=\sum_{e \in E^{+}(v)} y_{e}=$ $\sum_{e \in E_{+}(v)} \sum_{t=0}^{\theta} x_{e}(t)$. Therefore, constraint (2) holds for $\theta \geq t_{v}$. We have established that constraint (2) is obeyed.

By taking $\theta=T \geq t_{v}$ in the previous argument, we obtain that constraint (3) holds for all $v \in V_{*} \cap V_{y}$. For all sources $v \in V_{+}$incoming flow is zero: $\sum_{e \in E_{-}(v)} \sum_{t \in \mathbb{T}} x_{e}(t)=0$, since no edges enter a source. On the other hand, outgoing flow equals supply: $\sum_{e \in E_{+}(v)} \sum_{t \in \mathbb{T}} x_{e}(t)=$ $\sum_{e \in E_{f}(v)} y_{e}=-d_{v}$. Therefore, constraint (3) holds for all sources. The proof for sinks is similar, taking into account no edges exit sinks. Therefore, constraint (3) is obeyed.

Having proven that $x$ is a flow, it is easy to see that it is feasible, since $0 \leq x_{e}(t) \leq y_{e} \leq u_{e}$. Finally, $\varphi(x)=\sum_{e \in E} \sum_{t \in \mathbb{T}} \varphi_{e}\left(x_{e}(t), t\right)=$ $\sum_{e \in E_{y}} \varphi_{e}\left(x_{e}\left(t_{v}\right), t_{v}\right)=\sum_{e \in E} \varphi_{e}^{0}\left(y_{e}\right)=\varphi^{0}(y)$.

In the above proof we employ the fact that $T=+\infty$ only to maintain that $t_{v} \leq T, \forall v \in V_{y}$. However, if we denote by $|L|=\sum_{e \in L} \tau_{e}$ the time-length of a path in $\mathcal{N}$, then we immediately obtain that $t_{v} \leq$ $\max _{L \in \mathcal{N}}\{|L|\}$. Hence $\max _{L \in \mathcal{N}}\{|L|\}$ is an upper bound for the makespan 
of flow $x$ as constructed in the above lemma, and we can broaden the class of networks we examine.

Lemma 3. Let $\mathcal{N}$ be a dynamic network with cost functions constant in time such that $T \geq \max _{L \in \mathcal{N}}\{|L|\}$. If $y$ is a static flow in $\mathcal{N}^{0}$ such that its base graph $\mathcal{G}_{y}$ is a forest, then there exists a dynamic flow $x$ in $\mathcal{N}$ such that $\varphi(x)=\varphi^{0}(y)$.

To make the connection between Lemma 1, Lemma 3, and minimumcost flows in dynamic networks, we will employ the following property of minimum-cost flows in static networks with concave cost functions $[8,9]$.

Lemma 4. Let $\mathcal{N}^{0}$ be an uncapacitated static network with concave nondecreasing cost functions. If there exists a flow in $\mathcal{N}^{0}$, then there exists a minimum-cost flow $y$ in $\mathcal{N}^{0}$ such that its base graph $\mathcal{G}_{y}$ is a forest.

We are now able to prove the main result of this sub-section. Denote by $y^{T}$ the dynamic flow in $\mathcal{N}$ obtained from a forest-like flow $y$ in $\mathcal{N}^{0}$ such that $y_{(v, w)}^{T}(t)=y_{(v, w)}$ if $t=t_{v}$, and $y_{(v, w)}^{T}(t)=0$ otherwise, where $t_{v}$ are defined as in (5).

Theorem 1. Let $\mathcal{N}$ be an uncapacitated dynamic network with cost functions concave with regard to flow and constant in time such that $T \geq \max _{L \in \mathcal{N}}\{|L|\}$. If there exists a flow in $\mathcal{N}$, then there exists a minimum-cost forest-like flow $z$ in $\mathcal{N}^{0}$, and the flow $z^{T}$ is a minimumcost flow in $\mathcal{N}$.

Proof. Since there exists a flow in $\mathcal{N}$, a flow can be constructed in $\mathcal{N}^{0}$ according to Lemma 1 . Hence, according to Lemma 4 there exists a minimum-cost forest-like flow in $\mathcal{N}^{0}$; denote this flow by $z$. Flow $z^{T}$ is a minimum-cost flow in $\mathcal{N}$. Indeed, for any flow $x$ in $\mathcal{N}$ we have $\varphi\left(z^{T}\right)=\varphi^{0}(z) \leq \varphi^{0}(y) \leq \varphi(x)$, where $y$ is a static flow in $\mathcal{N}^{0}$ such that $y_{e}=\sum_{t \in \mathbb{T}} x_{e}(t)$. Equality $\varphi\left(z^{T}\right)=\varphi^{0}(z)$ follows from Lemma 3, inequality $\varphi^{0}(z) \leq \varphi^{0}(y)$ from the fact that $z$ is a minimum-cost flow in $\mathcal{N}^{0}$, and inequality $\varphi^{0}(y) \leq \varphi(x)$ from Lemma 1 .

Therefore, a dynamic minimum-cost flow can be computed using the following procedure: (i) find a forest-like minimum-cost flow $z$ in the corresponding static network; (ii) compute the constants $t_{v}$ and construct the dynamic minimum-cost flow $z^{T}$.

\subsection{An algorithmic approach}

We will present a combinatorial algorithm for the minimum-cost flow problem on dynamic networks that meet the conditions of Theorem 1 , and have exactly 1 source, based on the approach in [9]. We begin by 
examining networks with 1,2 , or 3 sinks, and then present an algorithm for the general case with $k$ sinks. Without loss of generality we will label the source node 0 , and the $k$ sinks $0,1, \ldots, k$.

We seek a minimum-cost flow with a base graph that is a forest. Since there is exactly 1 source, and $\sum d_{v}=0$, it follows that the base graph is connected, and thus it is a tree. Furthermore, since there are no edges entering sources or exiting sinks, the base graph rooted at the source will have the sinks as leaves; the problem becomes related to network design problems and Steiner trees [10].

One sink. We seek a rooted tree $\mathcal{G}_{y}$ with one leaf, in other words a simple path from the source 0 to sink 1 . Obviously the flow on any edge of the path is equal to the total demand $d_{1}$, and we need to minimize $\sum_{e \in \mathcal{G}_{y}} \varphi_{e}^{0}\left(d_{1}\right)$. This can be achieved by finding a shortest path from 0 to 1 with regard to edge lengths $\varphi_{e}^{0}\left(d_{1}\right)$. The coefficients $t_{v}$ for reconstructing the dynamic flow can be computed trivially by parsing the path from source to sink.

Two sinks. We seek a tree with fixed root 0 (the source) and two fixed leaves 1 and 2 (the sinks). Any such tree would have the structure of one of the trees in Figure 1, where by arrows we have denoted either simple paths or edges. Moreover tree structure (a) contains the other tree structures as degenerate cases; hence we can assume all trees have this structure. Denote by $d_{W}$ demand $\sum_{v \in W} d_{v}$; then flow on the paths from 0 to $w, w$ to 1 , and $w$ to 2 equals $d_{12}, d_{1}$, and $d_{2}$ respectively.

Consequently, we need to minimize for all $w \in V$ flow cost $\varphi^{0}(y)=$ $\sum_{e \in L_{0 w}} \varphi_{e}^{0}\left(d_{12}\right)+\sum_{e \in L_{w 1}} \varphi_{e}^{0}\left(d_{1}\right)+\sum_{e \in L_{w 2}} \varphi_{e}^{0}\left(d_{2}\right)$. This can be achieved with 3 one-to-all shortest path computations. Denote by $c_{v w}^{W}$ the minimum distance from $v$ to $w$ with regard to edge lenghts $\varphi^{0}\left(d_{W}\right)$; we compute for all $w \in V c_{0 w}^{12}$ from 0 to $w, c_{w 1}^{1}$ backwards from 1 to $w$, and $c_{w 2}^{2}$ backwards from 2 to $w$. Then it is straightforward to find $\min _{w \in V}\left\{c_{0 w}^{12}+c_{w 1}^{1}+c_{w 2}^{2}\right\}$ and then to compute $t_{v}$ by parsing the resulting tree. Due to concavity path overlapping does not lead to invalid results; we will address this in the proofs for the general case.

Three sinks. We seek a tree with fixed root 0 and fixed sink leaves 1 , 2 , and 3 . There are more structures such trees could take, but all are degenerate or proper cases of the three structures depicted in Figure 2. To find a minimum-cost tree-like flow, we compute a minimum-cost flow tree for each structure, and then select the optimal one. Consider computing a minimum-cost flow tree that fits structure (c) from Figure 2. Its cost can be expressed as the sum of costs along tree branches, as in the case 
(a)

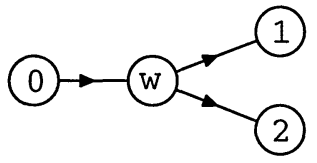

(b)

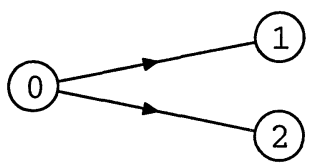

(c)

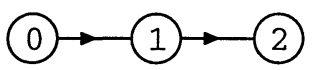

(d)

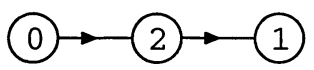

(a)

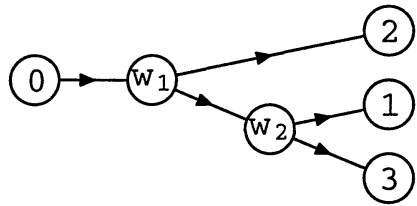

(b)

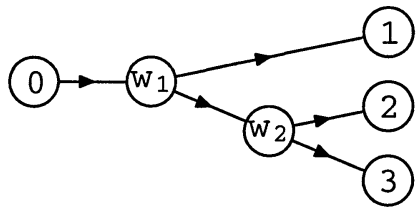

(c)

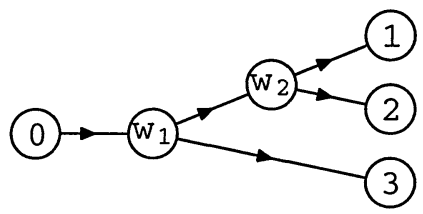

Figure 1. All structures for trees with one source and 2 sinks. Structure (b) is a degenerate case obtained from (a) by setting $w=0$; structure (c) is obtained from (a) by setting $w=1$; structure (d) is obtained from (a) by setting $w=2$.
Figure 2. All non-degenerate structures for trees with one source and 3 sinks. All other structures can be obtained as degenerate cases by using various combinations of setting $w_{1}$ and $w_{2}$ to $0,1,2,3$, and each other.

with two sinks. Therefore, the minimization can be achieved with $O(n)$ one-to-all shortest path computations. We compute $c_{v 1}^{1}, c_{v 2}^{2}, c_{v 3}^{3}, c_{0 v}^{123}$ for all $v \in V$, as well as $c_{v w}^{12}$ for all $v \in V$ and $w \in V$. Then we find $\min _{v, w \in V}\left\{c_{0 v}^{123}+c_{v w}^{12}+c_{v 3}^{3}+c_{w 1}^{1}+c_{w 2}^{2}\right\}$ and finally $t_{v}$. Minimum-cost trees for the other two structures are computed similarly.

The general case. We use dynamic programming to generalize, and present algorithm MINFLOW for computing the minimum cost of a dynamic flow in network $\mathcal{N}$ that satisfies the conditions of Theorem 1. We will consider given: (i) the dynamic network $\mathcal{N}$ with $n$ nodes, $m$ edges, 1 source, and $k$ sinks; (ii) the set of all tree structures with $k$ leaves $\mathbb{A}$.

Each structure $\mathcal{A}=\left(V_{\mathcal{A}}, E_{\mathcal{A}}\right) \in \mathbb{A}$ that fits a tree $G_{x}$ is represented itself as a tree that contains the root and all sinks in $G_{x}$. Moreover, $\mathcal{A}$ contains a new node for each node in $G_{x}$ that has more than one outbound edge; we will call these branch nodes. Tree $\mathcal{A}$ also contains a new edge for each simple path in $G_{x}$ consisting of nodes with exactly one outbout and exactly one inbound edge. 
The algorithm then calls MINTREE for each structure $\mathcal{A} \in \mathbb{A}$, which returns the minimum-cost of a flow with structure $\mathcal{A}$. The key element of MINTREE is the matrix $c[v][w]$, where $v \in V_{\mathcal{A}}$ and $w \in V$. The algorithm computes all elements of this matrix so that $c[v][w]$ equals the cost of sending $d[v]$ units of flow from node $w$ to the sinks that are in the subtree $\mathcal{A}_{v}$ of node $v$ so that to satisfy their demands. Here $d[v]=d_{v}$ for all sinks $v \in V_{-}$and $d[v]=\sum_{\alpha \in \mathcal{A}_{v} \cap V_{-}} d_{v}$ for all branch nodes. More loosely, $c[v][w]$ represents the cost of "assigning" branch node $v$ to network node $w$.

For all sinks $v \in V_{-}$the algorithm initializes $c[v][v]$ to 0 ; indeed the cost of getting the flow to $v$ from $v$ is zero. All other elements $c[v][w]$ with $v \in V_{-}$and $v \neq w$ are assigned $+\infty$. Then we are able to efficiently compute $c[v][w]$ by walking up the tree $\mathcal{A}$, and in the end obtain the sought minimum cost in element $c[0][0]$.

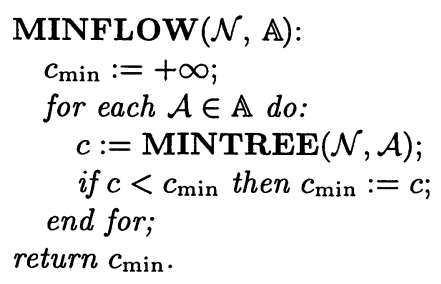

$\operatorname{MINLEN}(\mathcal{N}, v, l[]):$

return minimum distances $s[$ from $v$ to all nodes in the network $\mathcal{N}$ with regard to edge lenghts $l[$.

\section{$\operatorname{DESC}(\mathcal{A}, v)$ :}

return the set $E_{\mathcal{A}}^{+}(v)$ of all direct descendants of node $v$ in tree $\mathcal{A}$.

$\operatorname{READY}(\mathcal{A}, b[]):$

return the set $\left\{v \in V_{\mathcal{A}} \mid b[v]=0 \wedge b[w]=\right.$ $1, \forall w \in \operatorname{DESC}(\mathcal{A}, v)\}$ of all unprocessed nodes ready to be processed.

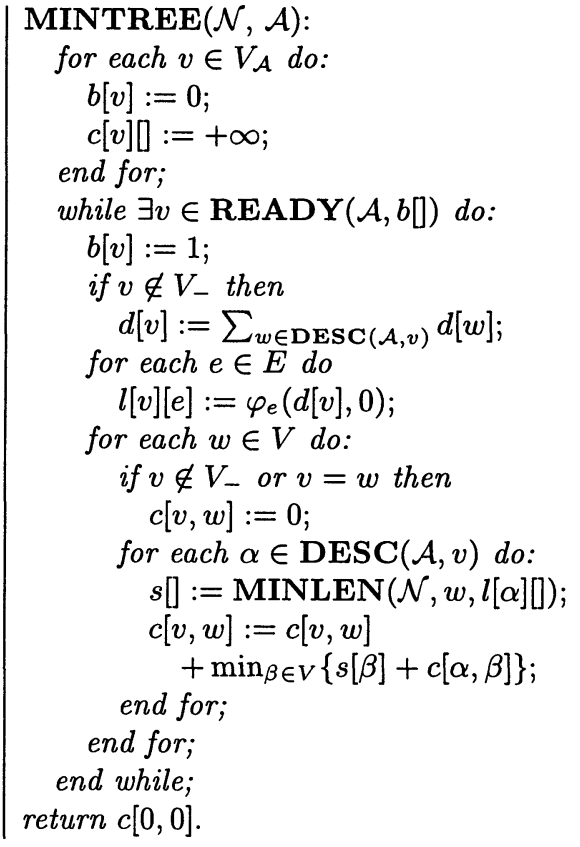

The algorithm can be modified to return not just the minimum cost, but also the flow for which it is obtained.

Lemma 5. Let $\mathcal{N}$ be an uncapacitated dynamic network with cost functions concave with regard to flow and constant in time such that $T \geq$ $\max _{L \in \mathcal{N}}\{|L|\}$. If there exists a flow in $\mathcal{N}$, then the cost computed by sub-algorithm $\operatorname{MINTREE}(\mathcal{N}, \mathcal{A})$ equals or exceeds the cost of at least one flow in $\mathcal{N}$. 
Proof. It results from the definition of READY and the use of vector $b$ that at the point when the algorithm begins computing $c[v][]$ for a non-sink node $v \in E_{\mathcal{A}}, c[w][$ is already computed for all descendants $w \in E_{\mathcal{A}}^{+}(v)$. It also holds that $d[v]$ is computed to equal the total demand of all sinks under $v$.

When computing $c[v][w]$, the algorithm sums for each descendant the cost of sending flow from that descendant plus the cost of getting the flow to that descendant. On the other hand, due to concavity, the sum of the costs of sending $\xi_{1}$ and $\xi_{2}$ units of flow across an edge $e$ equals or exceeds the cost of sending $\xi_{1}+\xi_{2}$ units together.

It follows by induction up the tree $\mathcal{A}$ that $c[v, w]$ equals or exceeds the cost of at least one way of sending $d[v]$ units of flow from node $w$ to the sinks in the sub-tree with root $v$ in $A$ while obeying flow conservation constraints and meeting sink demands. Therefore $c[0][0]$ equals or exceeds the cost of at least one way of sending $-d_{0}$ units of flow from source 0 to all the sinks while meeting their demands and obeying flow conservation constraints.

Lemma 6. Let $\mathcal{N}$ be an uncapacitated dynamic network with cost functions concave with regard to flow and constant in time such that $T \geq$ $\max _{L \in \mathcal{N}}\{|L|\}$. If there exists a minimum-cost tree-like flow $x$ with structure $\mathcal{A}$ in $\mathcal{N}$, the cost computed by $\operatorname{MINTREE}(\mathcal{N}, \mathcal{A})$ equals the minimum cost of a flow in $\mathcal{N}$.

Proof. If flow $x$ has structure $\mathcal{A}$ then we can associate to each node $v \in \mathcal{A}$ a node $v_{x} \in V$. We will refer to the cost of flow being transported on the edges of a sub-tree of the base graph $\mathcal{G}_{x}$ as the cost of that subtree.

We note that MINTREE computes the shortest path from a node to its descendants, and uses the minimum total cost selecting each descendant. It follows by induction up the tree $\mathcal{A}$ that $c\left[v, v_{x}\right]$ is less than or equal to the cost of the subtree rooted at $v_{x}$ with regard to flow $x$.

Therefore, $c[0][0] \leq \varphi(x)$. But according to Lemma $5, c[0][0]$ exceeds or equals the cost of a flow in $\mathcal{N}$. Since $y$ is a minimum-cost flow in $\mathcal{N}$ we obtain that $c[0][0]=\varphi(x)$.

Theorem 2. Let $\mathcal{N}$ be an uncapacitated dynamic network with cost functions concave with regard to flow and constant in time such that $T \geq \max _{L \in \mathcal{N}}\{|L|\}$. If there is a flow in $\mathcal{N}$, then algorithm MINFLOW computes the minimum cost of a flow in the dynamic network $\mathcal{N}$.

Proof. Since there is a flow in $\mathcal{N}$, there exists a minimum-cost flow $x$ in $\mathcal{N}$, and hence there is a structure $\mathcal{A} \in \mathbb{A}$ that fits it. Therefore, according to Lemma $6 \operatorname{MINTREE}(\mathcal{N}, A)$ will return the minimum cost of a flow in $\mathcal{N}$. Moreover, since according to Lemma 5 any cost returned by MINTREE equals or exceeds the cost of a flow, all other calls 
will return costs equal to or higher than the cost of the minimum flow. Since MINFLOW selects the minimum from all calls, it will return the minimum cost of a flow in $\mathcal{N}$.

\subsection{Algorithm complexity}

We first examine the complexity of sub-algorithm $\operatorname{MINTREE}(\mathcal{N}, A)$. With an appropriate choice of tree representation, the calls to READY and DESC do not impact the order of complexity given by the loop structure. We assume Dijkstra's algorithm is used for the MINLEN computations. From the loop structure of MINTREE it follows that its execution has the complexity $O\left(\left|V_{\mathcal{A}}\right|+\left|V_{\mathcal{A}}\right|(m+n(n \log n+m+n))\right)=$ $O(k n(n \log n+m))$. Therefore algorithm MINFLOW has complexity $O(|\mathbb{A}| k n(n \log n+m)$. Obviously, the number of tree structures in $\mathbb{A}$ is at least exponential of the number of leaves $k$. However, if we consider networks with $k$ fixed (not part of the input), then the algorithm is polynomial, with complexity $O\left(m n+n^{2} \log n\right)$.

\section{References}

[1] L. R. Ford, Jr. and D. R. Fulkerson. Constructing maximal dynamic flows from static flows. Operations Res., 6:419-433, 1958.

[2] L. R. Ford, Jr. and D. R. Fulkerson. Flows in networks. Princeton University Press, Princeton, N.J., 1962.

[3] D. Lozovanu and D. Stratila. The minimum-cost flow problem on dynamic networks and an algorithm for its solving. Bul. Acad. Ştiinţe Repub. Mold. Mat., (3):38-56, 2001.

[4] Lisa Fleischer and Martin Skutella. The quickest multicommodity flow problem. In Integer Programming and Combinatorial Optimization, pages 36-53. Springer, Berlin, 2002.

[5] Bettina Klinz and Gerhard J. Woeginger. Minimum cost dynamic flows: the series-parallel case. In Integer programming and combinatorial optimization (Copenhagen, 1995), pages 329-343. Springer, Berlin, 1995.

[6] James B. Orlin. Minimum convex cost dynamic network flows. Math. Oper. Res., 9(2):190-207, 1984.

[7] Lisa Fleischer and James B. Orlin. Optimal rounding of instantaneous fractional flows over time. SIAM J. Discrete Math., 13(2):145-153 (electronic), 2000.

[8] D. D. Lozovanu. Properties of optimal solutions of a grid transport problem with concave cost function of the flows on the arcs. Izv. Akad. Nauk SSSR Tekhn. Kibernet., (6):94-98, 1982.

[9] D. D. Lozovanu. Ekstremalno-kombinatornye zadachi i algoritmy ikh resheniya. "Shtiintsa", Kishinev, 1991.

[10] Frank K. Hwang, Dana S. Richards, and Pawel Winter. The Steiner tree problem. North-Holland Publishing Co., Amsterdam, 1992. 